\title{
Noch viele offene Fragen
}

\section{Vor kurzem legte die dänische EU-Präsidentschaft einen überarbeiteten Richtlini- envorschlag zum europäischen Emissionshandelssystem für Treibhausgase vor. Welche Dynamiken sind zu beobachten und welche kritischen Punkte zeichnen sich $a b$ ?}

I Von Sven Rudolph $m$ August diesen Jahres legte die dänische EUPräsidentschaft nach intensiver Diskussion einer früheren Fassung einen überarbeiteten Richtlinienentwurf zum europäischen Handel mit Treibhausgasemissionen ab 2005 vor. Ziel ist die kosteneffiziente Erfüllung der europäischen Kyoto-Verpflichtung einer Treibhausgasreduktion im Zeitraum 2008-2012 um acht Prozent gegenüber 1990. Die seit den 70er-Jahren von Ökonomen betonten und empirisch in den USA bestätigten Vorteile geographischer, technologischer und temporärer Flexibilität bei der Erfïllung von Minderungsanforderungen sollen realisiert und das ökologische Ziel effektiv erreicht werden. Gleichsam soll die Kompatibilität europäischer Regulierungen mit dem internationalen Regime gewährleistet werden, das gemäß dem Kyoto-Protokoll die Nutzung flexibler Mechanismen zur Erreichung von Klimaschutzzielen erlaubt.

Der EU-Richtlinienvorschlag umfasst grundsätzlich alle Treibhausgasemissionen, jedoch werden aus Praktikabilitätsgründen anfangs allein die Kohlendioxid-Emissionen einbezogen. Gemäß dem bestehenden EU-Burden-Sharing erhalten die Mitgliedsstaaten Kohlendioxid-Emissionslizenzen, welche die absolute Gesamtemissionsmenge pro Land fixieren. Dies geben die Nationalstaaten mittels eines genehmigungsbedürftigen, nationalen Allokationsplans zunächst kostenlos (Grandfathering) an die betroffenen Wirtschaftssektoren bzw. Anlagen aus. Von der Richtlinie betroffen sind ca. 5000 Industrieanlagen der Energiewirtschaft, der Eisenmetall-, der Papier- und Zellstoff- sowie der mineralverarbeitenden Industrie. Gemeinsam verursachen diese ca. 46 Prozent der europäischen Kohlendioxidemissionen.

Entgegen der ursprünglich vorgesehenen verpflichtenden Teilnahme können die Nationalstaaten gemäß dem aktuellen Vorschlag bestimmte Anlagen oder Branchen bis einschließlich 2007 vom Emissionshandelssystem freistellen, wenn andere nationale Maßnahmen nachweisbar dem Emissionshan- del entsprechende Emissionsreduktionen bei analogen Monitoringverfahren und Sanktionsmaßnahmen garantieren $(O p t-O u t)$. Zudem betont der aktuelle Vorschlag nun, entgegen der früheren Idee einer separaten Richtlinie, explizit die Erwünschtheit einer zeitnahen Integration der projektbezogenen Mechanismen Joint Implementation (JI) und Clean Development Mechanism (CDM) in den EUEmissionshandel und sichert die Anerkennung von Emissionskrediten aus diesen Projekten zu, wobei die genauen Anforderungen hierfür bis zum Start des Emissionshandels im Jahr 2005 erst noch festgelegt werden müssen.

\section{- Kritische Punkte}

Der nationale Allokationsplan wird politisch brisant durch seine erheblichen verteilungspolitischen, ökologischen und ökonomischen Auswirkungen.

Hinsichtlich der Lizenzzuteilungsbasis werden im wesentlichen drei Varianten diskutiert:

1. Ein Benchmarking mit der Festlegung spezifischer, beispielsweise anlagenindividueller Emissionswerte würde den ökologisch sinnvollen Brennstoffwechsel als Vermeidungsoption behindern.

2. Ein reines Grandfathering auf der Basis der Emissionen von 1990/92 hingegen honoriert Stilllegungen von Anlagen in der Vergangenheit.

3. Die Wahl eines späten Basisjahres wiederum lässt Investitionen in Emissionsvermeidungen in der Vergangenheit (Early Action) unberücksichtigt. Diese könnten jedoch in letzterer Variante explizit gegenüber dem Staat nachgewiesen und gegebenenfalls berïcksichtigt werden.

Die Nutzung von in meist bilateralen, internationalen JI- und CDM-Projekten erworbenen, transferierbaren Emissionskrediten im EU-Handelssystem eröffnen zwar erhebliche Kosteneinsparpotenziale. Es droht aber die Gefahr, dass die ökologische Integrität durch den Import von Emissionskrediten beispielsweise aus fragwürdigen Senkenprojekten aufgeweicht wird. Strenge ökologische Anforderungen sind notwendig, um dies zu verhindern.
Das Zusammenwirken des EU-Emissionshandels mit nationalen Maßnabmen ist umstritten. Die Selbstverpflichtungserklärung der deutschen Wirtschaft könnte durch den europäischen Emissionshandels überflüssig werden. Da der Emissionshandel keinen spezifischen Vorteil für erneuerbare Energien aufweist, ist eine Aufrechterhaltung des Erneuerbare-Energien-Gesetzes geboten. Im Bereich der Kraft-Wärme-Kopplung (KWK) als ökologisch sinnvolles Verfahren sind im Richtlinienvorschlag explizite Regelungen zu ergänzen, die eine Benachteilung der KWK verhindern. Hinsichtlich des Zusammenspiels von Öko-Steuer und Emissionshandel können die für das produzierende Gewerbe existierenden Ausnahmeregelungen für diejenigen Anlagen beibehalten werden, die am Emissionshandel teilnehmen. Nicht-teilnehmende Anlagen würden hingegen beispielsweise mit dem regulären Steuersatz belastet, um gesamtgesellschaftliche Wettbewerbsgerechtigkeit herzustellen.

Die Freiwilligkeit der Teilnahme am Emissionshandelssystem, das heißt ein Opt-Out für bestimmte Anlagen oder Branchen, verhindert einen funktionierenden Markt, da allein Lizenzanbieter teilnehmen würden. Positive Anreize zur Teilnahme in Form von Subventionen erscheinen aus Kostengründen wenig sinnvoll. Allein negative Anreize für Nichtteilnehmer beispielsweise in Form drohender anderer staatlicher Maßnahmen zur Erreichung der dann individualisierten Emissionsminderungsziele wären vorstellbar. In einem solchen Fall käme es bei Freiwilligkeit der Teilnahme zu einem Wettbewerb der Instrumente. Abschließend bleibt zu hoffen, dass die weiteren Diskussionen nicht zu einer Verwässerung der ökologischen Effektivität des EU-Emissionshandels führen. Die Lizenzlösung wird auch in Zukunft nur dann eine wichtige Rolle in der praktischen Umweltpolitik spielen können, wenn die Ausgestaltung eines Emissionshandelssystems neben ökonomischen und politischen auch ökologischen Anforderungen gerecht wird.

\section{Der Autor}

Sven Rudolph promoviert am Fachbereich Wirtschaftswissenschaften der Universität Kassel zum Thema und ist zudem für den Bund für Umwelt und Naturschutz tätig.

Kontakt: E-Mail: rudolph@student.uni-kassel.de 
(c) 20I0 Authors; licensee IÖW and oekom verlag. This is an article distributed under the terms of the Creative Commons Attribution Non-Commercial No Derivates License (http://creativecommons.org/licenses/by-nc-nd/3.o/), which permits unrestricted use, distribution, and reproduction in any medium, provided the original work is properly cited. 